\title{
La poca penetrazione della dialisi peritoneale in Italia (ovvero la continua commiserazione dei peritonealisti)
}

\author{
Emilio Giulio Galli \\ Direttore U.O. Nefrologia dell'Ospedale di Treviglio, Treviglio (BG)
}

\begin{abstract}
The LOW PENETRATION OF PERITONEAL Dialysis IN ITALY
Abstract. The low penetration of peritoneal dialysis (PD) in Italy is determined by multiple factors, among which the lack of acceptance of this dialysis method by many nephrologists, as well as the large number of centers with few PD patients, which do not allow for the creation of a competent experience. Not less importantly, there is a lack of planning and guidance by the institutions.
\end{abstract}

Key words: Chronic renal failure, Peritoneal dialysis, Health planning

Conflict of interest: None.

Financial support: None.

Accettato: 28 Agosto 2013

Da anni si discute, si scrive e si organizzano convegni sul perché la dialisi peritoneale (DP) in Italia non cresce. Tutta questa discussione è, però, fatta quasi esclusivamente da chi la dialisi peritoneale la utilizza. Da anni, nelle assemblee del Gruppo di Studio della Dialisi Peritoneale, durante i congressi nazionali della SIN e nei convegni di DP, incontro sempre le stesse persone che si pongono sempre la stessa domanda: ma perché la DP in Italia è ferma da anni al 10\%?

Ritengo che, oggi, siano i nefrologi che lavorano in centri dove non viene utilizzata la DP a dover rispondere a questa domanda.

Ma perché la DP, oggi, non esiste in tutti i centri di nefrologia? Siamo ancora negli anni '80, quando Shaldon la definiva una metodica di seconda classe, per pazienti di seconda classe, fatta da medici di seconda classe? Dopo 30 anni di produzione scientifica comprovante la pari efficienza, per almeno 5-6 anni, con la sorella emodialisi, si mantengono ancora vive nei fatti le affermazioni di Shaldon? Sono, forse, Maiorca, Buoncristiani, La Greca e Cancarini medici di seconda classe? I 35 pazienti in DP nel mio centro sono stati messi in DP perché sono di seconda classe?

Ritengo, invece, che un peritonealista sia un nefrologo dotato di competenza specifica, esperienza spesso fatta sul campo (molte scuole di specializzazione in Italia hanno solo un minimo programma didattico di DP) e motivazioni particolari. Vi è ancora un atteggiamento di diffidenza nello staff medico verso la DP anche nei centri dove la si pratica. Quante volte vengono segnalati al peritonealista del centro dei pazienti solo perché non idonei all'emodialisi?

Ovviamente, la mancata crescita della DP non è attribuibile solo alla mancata accettazione da parte dei nefrologi di questa metodica dialitica. La poca maturazione dei nefrologi verso la DP è dovuta anche alla mancanza di strutture adatte (sempre, però, disponibili per l'emodialisi), alla carenza di personale (la DP è la metodica che impiega meno personale) e al grosso numero di centri con meno di 10 pazienti in DP che non permettono quell'esperienza fatta sul campo citata prima.

Penso che anche l'organizzazione di un centro DP abbia la sua importanza. Sono a conoscenza di centri dove non vi è personale medico e infermieristico dedicato alla DP. Il fatto che tutti si occupino di tutto, può generare una scarsa motivazione, poca specializzazione e poca esperienza. Questi sono ottimi fattori per il fallimento di una metodica. Nel 2003, i dati del censimento della DP del GdSDP mostrano che, in 230 centri (su 237 centri totali), si sono occupati della metodica 340 medici e 503 IP. Considerando che in più del $50 \%$ dei centri non vi è personale dedicato alla DP, è evidente che i 4328 pazienti erano seguiti da personale medico e infermieristico diverso che, molto probabilmente, si dedicava anche alla nefrologia e all'emodialisi.

Ovviamente, vi sono anche problematiche economiche. Le tariffe per il rimborso della DP sono inferiori rispetto all'emodialisi e i centri privati non hanno convenienza a trattare $\mathrm{i}$ pazienti con la DP. Queste problematiche sono puntualmente ributtate sul tavolo della discussione ogni volta che ci si pone il quesito: perché non cresce la DP? Indubbiamente, sono fat- 


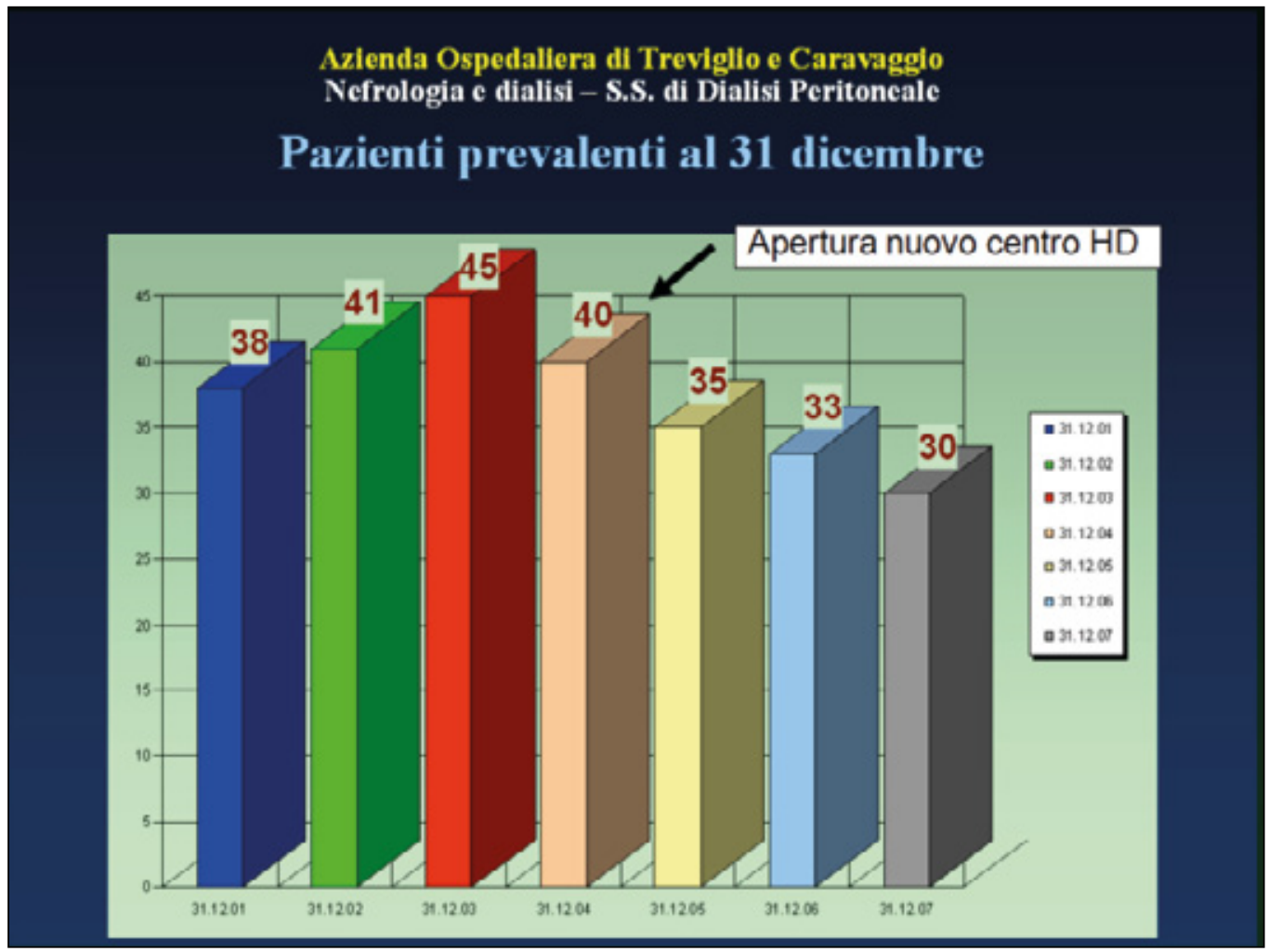

Fig. 1

Azlenda Ospedallera di Trevigllo e Caravagglo

Nefrologia e dialisi - S.S. di Dialisi Peritoneale

Pazienti prevalenti al 31 dicembre

tori molto importanti, che, sicuramente, hanno condizionato la poca crescita della DP. Ma, allora, perché Silvio Bertoli dirige una nefrologia in una struttura privata e ha in terapia pazienti in DP da anni? Perché la DP a Treviglio con 46 pazienti incidenti nel 2005 ha "prodotto" 530.000 euro di attivo (secondo margine operativo)? Perché, in una regione con bassa penetrazione della DP (Sicilia), Flavia Caputo aveva, al 31-12-2004, 46 pazienti? Perché, in una regione del sud, Roberto Corciulo tratta quasi lo stesso numero di pazienti del "nordico" Claudio Ronco?

Ma è solo il sud Italia che fa drasticamente calare la percentuale di penetrazione della DP?

A questa domanda rispondo con dei dati della nordica provincia di Bergamo:

- abitanti: 1.100 .000 circa

- aziende ospedaliere che effettuano trattamenti dialitici: 6 (3 private e 3 pubbliche, di cui una ha dato in outsourcing la nefrologia)

- aziende ospedaliere che offrono la DP: 2 (Treviglio e Bergamo)

- pazienti dializzati in provincia: circa 800

- pazienti in DP: circa 80

I due centri che effettuano la DP in provincia hanno dato disponibilità a trattare $\mathrm{i}$ pazienti in DP anche dei centri che non la effettuano. Pazienti inviati da questi ultimi centri: 0 .

Questi esempi penso siano delle risposte chiare a tante giustificazioni economiche superabili dalla volontà. La stessa volontà che sembra mancare anche a livello politico. Nei piani sanitari nazionali e regionali sembra di riconoscere la calligrafia di qualche nefrologo emodialista. Frequentemente, viene trattata la DP marginalmente, senza fornire disposizioni chiare e senza incentivazione. I nefrologi lombardi si ricorderanno bene cosa erano gli incentivi per l'emodialisi nei centri di assistenza limitati (CAL) negli anni pre-crisi. Se vi fosse un minimo di incentivazione economica verso il personale, $\mathrm{i}$ partner dei pazienti (come avviene in Piemonte) e le residenze per anziani, sicuramente la colonnina dei pazienti in DP nei grafici dei registri di dialisi aumenterebbe.

Gli assessori regionali sanno della maggiore economicità della DP?

La stessa Società Italiana di Nefrologia, per anni, non ha dimostrato un grande interesse per la DP.

Nel censimento dei centri italiani del 2006, nei 6 moduli predisposti per la raccolta dati, in uno solo vi era una sola domanda riguardante la DP: al 31-12-2005 quanti pazienti in DP?

Le ditte del nostro settore stanno aprendo nuovi centri di nefrologia e dialisi attraverso la formula "service" o creando delle fondazioni onlus a partecipazione mista pubblica-privata. Questa situazione, sicuramente positiva, crea, però, la necessità "per contratto" di una completa saturazione dei posti in emodialisi. È altamente improduttivo lasciare dei posti tecnici vuoti in emodialisi e, di conseguenza, la scelta della metodica dialitica da parte del paziente è più "guidata" verso l'emodialisi. La maggiore disponibilità di posti in emodialisi ritengo sia determinante per spostare i nuovi ingressi in dialisi verso la metodica extracorporea, indipendentemente dal fatto che tali nuovi ingressi siano early referral o late referral. Nella Figura 1 , sopra riportata sono mostrati i pazienti prevalenti in DP al 
31 Dicembre di diversi anni nel mio centro. Da notare, nel 2004 , l'apertura di un nuovo centro di emodialisi in "service" e la conseguente riduzione dei pazienti in DP. Puro caso?

L'invecchiamento della popolazione e la sempre minore disponibilità dei partner potrebbero essere, ancora di più nei prossimi anni, un ulteriore ostacolo all'incremento della DP. Di contro, sono convinto che, di fronte a costi sanitari sempre più elevati e a una disponibilità di personale e di risorse sempre più bassa, una metodica dialitica di prima classe, per pazienti di prima classe e fatta da medici di prima classe, che comporti costi minori e che utilizzi meno personale, come lo è la DP, possa essere una risposta ai succitati problemi.

\section{Riassunto}

La poca penetrazione della Dialisi Peritoneale (DP) in Italia è tuttora dovuta a molteplici fattori, come la mancata accettazione, da parte di molti nefrologi, di questa metodica dialitica e il grosso numero di centri con pochi pazienti in DP, che non permette la formazione di un'efficiente esperienza. Non di secondaria importanza, una carenza di programmazione e di indicazioni da parte degli organismi istituzionali.
Parole chiave: Insufficienza renale cronica, Dialisi peritoneale, Programmazione sanitaria

Dichiarazione di conflitto di interessi: L'Autore dichiara di non avere conflitto di interessi.

Contributi economici degli autori: L'Autore dichiara di non aver ricevuto sponsorizzazioni economiche per la preparazione dell'articolo.

Indirizzo degli Autori:

Dr. Emilio Giulio Galli

Direttore U.O. Nefrologia

A.O. di Treviglio

P.le Ospedale 1

24047 Treviglio (BG)

emilio_galli@ospedale.treviglio.bg.it 